\title{
The changing face of childhood meningitis
}

$\mathrm{C}$ ANADIAN PEDIATRICIANS AND FAMILY PHYSICIANS WILL likely remember 1993 as the year when infections caused by Haemophilus influenzae type b (Hib) virtually disappeared. This remarkable development reflects the outstanding efficacy of universal infant vaccination programs initiated in all provinces during 1992. Their effect on Hib disease was swift: by the third quarter of 1993, the Canadian Paediatric Society's Immunization Monitoring Program, ACTive (IMPACT) network (1) reported a 90\% decline in preventable cases at the 10 participating pediatric centres, compared with the same period in 1992. The actual number of preventable cases encountered in the third quarter of 1993 was only three. These data understate the actual improvement over baseline conditions because other Hib vaccines used since 1985 had some impact on illness rates. Currently used Hib vaccines have an excellent safety record and rarely fail to protect after completion of the three-dose primary series.

The direct benefits of Hib disease eradication include an overall decrease in childhood meningitis cases of approximately $70 \%$ (2), along with virtual elimination of epiglottitis cases, and a substantial decrease in cases of septic arthritis, cellulitis, pneumonia and bacteremia. The indirect benefits include a refocusing of investigative effort on the second tier of serious bacterial pathogens affecting children, catalysed by the many technological insights learned from $\mathrm{Hib}$ and by the joy of conquering a serious pathogen.

The spotlight has already shifted. A renewed effort to control pneumococcal infections in children is well under way. Pneumococci are the leading cause of bacteremia and, with control of Hib, are now the number one cause of meningitis. Compared with Hib meningitis,

All material presented in Pediatric Infectious Disease Notes has been reviewed and approved by the chairperson, Canadian Paediatric Society Board and representative members of the Canadian Paediatric Society Committee on Infectious Disease and Immunization

Correspondence: Infectious Disease and Immunization Committee, Canadian Paediatric Society, 401 Smyth Road, Ottawa, Ontario K1H 8L1. Telephone (613) 737-2728, Fax (613) $737-2794$ pneumococcal cases are six times more likely to end fatally ( $19 \%$ case fatality) (2). In a Canadian case series (3) $56 \%$ of survivors had neurological sequelae. The recent increase in prevalence of pneumococci resistant to penicillin and multiple other antibiotics (4) has added a note of urgency to the development of improved vaccines suitable for use in infants. Multivalent, polysaccharide-protein conjugate vaccines look promising for use in infants to prevent otitis media and pneumonia, as well as invasive infections including meningitis.

One wonders if the recent, aggressive approach to controlling meningococcal outbreaks with communitywide vaccination programs is not conditioned in part by the success with $\mathrm{Hib}$, with a concomitant decrease in tolerance for life-threatening infections.

If the sun has set on Hib, it has clearly risen on group B streptococcal (GBS) infections. Major programs for peripartum chemoprophylaxis have been advocated (5), although many practical issues remain to be resolved. Detection of GBS-colonized women before delivery poses challenges, especially where culture facilities are limited or women have little or no prenatal care. Additionally, the cost of cultures and ensuring that results are available in the case room when needed are problematic. Only a small proportion of GBS-colonized women will deliver infants at high risk for invasive infection, but algorithms that attempt to restrict chemoprophylaxis according to existing risk factors work imperfectly. Late-onset GBS cases are most likely to present with meningitis and may not be prevented by chemoprophylaxis because the source of infection is often nonmaternal. The better long term solution will likely prove to be maternal immunization, using polysaccharide-protein conjugate vaccines (6). Such vaccines are under development, replacing candidate polysaccharide vaccines that proved to be insufficiently immunogenic.

Progress can have unfortunate effects as well. Just as dexamethasone treatment was becoming widely used for Hib meningitis, the target disappeared. Its usefulness in cases caused by pneumococci or meningococci is unproved. No data are available regarding its use in babies with GBS or other causes of neonatal meningitis. The low frequency of meningitis caused by these 
second-tier pathogens will make it difficult to reevaluate the usefulness of dexamethasone adjunctive treatment, leaving clinicians uncertain whether to use it.

\section{REFERENCES}

1. Scheifele D, Gold R, Duclos P and members of the Canadian Pediatric Society's Immunization Monitoring Program, Active (IMPACT). An innovative hospital-based national network for active surveillance of vaccine-associated adverse events, vaccine failures and selected infections in children. Proceedings of the 33rd Interscience Conference of Antimicrobial Agents and Chemotherapy, New Orleans, October 1993. (Abstract 1649)

2. Wenger JD, Hightower AW, Facklam RR, Gaventa S, Broome CV and the Bacterial Meningitis Study Group. Bacterial meningitis in the United States, 1986: Report of a multistate surveillance study. J Infect Dis
1990;162:1316-23.

3. Laxer RM, Marks MI. Pneumococcal meningitis in children. Am J Dis Child 1977;131:850-3.

4. Appelbaum PC. Antimicrobial resistance in Streptococcus pneumoniae: An overview. Clin Infect Dis 1992;15:77-83.

5. Committee on Infectious Diseases and Committee on Fetus and Newborn, American Academy of Pediatrics. Guidelines for prevention of group B streptococcal infection by chemoprophylaxis. Pediatrics 1992;90:775-8.

6. Wessels MR, Paoletti LC, Rodewald AK, et al. Stimulation of protective antibodies against type $1 \mathrm{a}$ and $1 \mathrm{~b}$ group B streptococci by a type 1a polysaccharide-tetanus toxoid conjugate vaccine. Infect Immun 1993;61:4760-6.

David Scheifele MD Vaccine Evaluation Center $B C$ 's Children's Hospital Vancouver, British Columbia

\section{ADULT INFECTIOUS DISEASE NOTES}

\section{Systemic antifungal drugs: Are we making any progress?}

$\mathrm{T}$ HE INCIDENCE OF OPPORTUNISTIC FUNGAL INFECTION IS increasing worldwide, and invasive fungal infections have become an important problem in patients with profound immunosuppression. Increased use of potent cytotoxic drugs and broad-spectrum antibacterial agents, as well as the AIDs pandemic, have been the main contributors in modifying the current epidemiological and clinical pictures of serious fungal infections. For years amphotericin B was the only antifungal drug available. Its administration was, however, plagued by important side effects and toxicities. Clinical tolerance is often achieved only through premedication with antipyretics, diphenhydramine or hydrocortisone. Electrolyte imbalance and renal toxicity are frequently observed. Finally, amphotericin B is often administered for long periods of time at high sustained doses, particularly in invasive infections, and can only be administered intravenously. It is therefore not surprising that alternatives have been sought during the past two decades, particularly to develop wide spectrum, nontoxic, well tolerated and easy to administer antifungal drugs.

Flucytosine was licensed in the early '70s. Its spec- trum of activity is narrow, including only strains of Candida species and Cryptococcus neoformans, while most other fungi are resistant or vary in their susceptibilities (1). Despite excellent absorption after oral administration, and good diffusion and tissue penetration, flucytosine has two important problems: emergence of resistance and serious toxicities. Drug resistance arising during flucytosine monotherapy is usually profound and accompanied by clinical deterioration (2). This emergence of secondary resistance led to recommendations to use flucytosine in combination with amphotericin B (2-3). Toxicities associated with flucytosine, ie, bone marrow aplasia and gastrointestinal toxicities, are common. Their presence correlates with high flucytosine serum levels (4). The elimination of flucytosine is mainly renal, and amphotericin B combined with flucytosine can cause sufficient renal impairment to decrease the elimination and increase the serum concentration of the latter, thus causing flucytosine-related toxicities. Leukopenia and diarrhea are also commonly associated with the use of flucytosine. In immunocompromised hosts, such as AIDS patients, these phenomena are commonly observed. 
Despite a high incidence of cryptococcal and candidal infections in these patients, the use of flucytosine is problematic.

The discovery of the imidazoles represented an important therapeutic advance for the systemic antifungal drug armamentarium. Miconazole was approved in the United States (but not in Canada) for intravenous use against systemic fungal infections. It was found to have significant toxicities, and its present role is limited to Pseudoallecheria boydii infections. Ketoconazole was the first systemic azole of importance to be commercialized. Its oral administration and absorption was originally perceived as an important advantage over amphotericin B. Oral absorption, however, varies considerably among individuals (5). Histamine-2 blocking agents and antacids drastically reduce blood levels of ketoconazole when administered concomitantly (6). Although this can be reversed by the administration of acidic food, it seems inappropriate in patients in need of antiacid medications. Several drug interactions have also been reported with ketoconazole. Of importance is the cardiotoxicity of the antihistaminic drugs terfanadine and astemizole when administered concurrently with ketoconazole (7). Cyclosporine blood concentrations are increased in patients treated with ketoconazole, which adds up to cyclosporine's potential for nephrotoxicity. Rifampin accelerates the hepatic metabolism and lowers the blood levels of ketoconazole. Finally, ketoconazole causes a dose-dependent depression of serum testosterone and adrenocorticoid hormone (ACTH)-stimulated cortisol response. The efficacy of ketoconazole has been documented in the treatment of dermatophyte infections and endemic fungal infections such as histoplasmosis, blastomycosis, paracoccidioidomycosis and coccidioidomycosis. It is of no value in the treatment of cryptococcal infections, aspergillosis or mucorales infections. The role of ketoconazole in the management of fungal infections in severely immunocompromised patients has remained quite limited. Over the years, clinicians have used ketoconazole to treat neutropenic or AIDS patients with oral and esophageal candidiasis successfully. Associated toxicities, the uncertain degree of absorption and the drug interactions have, however, limited ketoconazole use.

Fluconazole alleviated some of those concerns. Fluconazole is water soluble and can be administered orally or intravenously. Given orally it is essentially completely absorbed, and its absorption is independent of gastric $\mathrm{pH}$. Hepatic degradation through enzymes induced by drugs such as rifampin occurs to a lesser degree with fluconazole than with ketoconazole (8). Cyclosporine plasma levels seem to be increased only by high doses of fluconazole (ie, greater than 400 $\mathrm{mg}$ /day). Clinically the efficacy of fluconazole has been slightly inferior to that of amphotericin B in the primary treatment of acute cryptococcal meningitis in AIDS patients (9). It has, however, emerged as the drug of choice for maintenance therapy (10). Evidence of the efficacy of fluconazole in the treatment of chronic disseminated candidiasis is slowly emerging (11). In some studies, the prophylactic use of fluconazole has, however, been associated with a selection of naturally resistant Candida krusei and Candida glabrata isolates, particularly in severely compromised bone marrow transplant patients and in leukemic patients (12-13). One may disregard this observation because determination of in vitro sensitivity of yeasts to azoles is particularly difficult to perform. Yeasts are never completely inhibited in vitro, where their degree of inhibition is not related linearly to the drug concentrations used. The minimal inhibitory concentration end-point cannot, therefore, be determined with precision. Both treatment failure and yeast superinfections due to resistance have, however, been reported in patients treated with fluconazole, suggesting a certain clinical relevance with the in vitro observations (14). Whether these isolates represent natural or acquired resistance is of concern based on the well established experience of the emergence of bacterial resistance associated with the use of antibacterial agents.

Itraconazole was recently licensed in Canada. It has a much broader spectrum of activity than the former azoles, particularly for the filamentous fungi such as Aspergillus. Despite several similarities to ketoconazole, early studies have shown a better safety profile due to a greater affinity and specificity to fungal cytochrome P450 than to human enzymes. Mild gastrointestinal reactions are the most commonly observed adverse reactions. Other reactions, occurring in fewer than $10 \%$ of patients, include hypertriglyceridemia, hypokalemia and mild liver enzyme elevations (15). Like ketoconazole, however, concurrent administration of itraconazole with antihistaminic drugs may result in life-threatening cardiac dysrhythmias. In vitro data suggest that the interaction of itraconazole will produce a less pronounced effect on the prolongation of the QT interval than the ketoconazole-astemizole combination. Like ketoconazole, itraconazole requires an acid gastric $\mathrm{pH}$ for optimal absorption. Itraconazole has demonstrated efficacy against a broad range of mycotic infections, notably aspergillus infections. Current information indicates that itraconazole has been effective in 46 to $65 \%$ of patients with invasive aspergillosis (16). Itraconazole seems to offer an alternative as salvage therapy in patients with aspergillus infections who have failed to respond to amphotericin B (17). Itraconazole may also be useful in patients with the more chronic form of disseminated aspergillosis. In these patients prolonged therapy is recommended. Itraconazole can then be used on an out-patient basis, but its clinical efficacy in this particular situation needs to be documented further. The role of itraconazole in the treatment of candidiasis in granulocytopenic patients is still unclear, due to the limited number of available studies. Results however, appear promising and itraconazole 
has shown efficacy similar to that of fluconazole and ketoconazole (18-19). Absorption of oral drugs is dependent on the integrity of the gastrointestinal tract. Gastrointestinal mucosa altered by chemotherapy occurs frequently in neutropenic patients and interferes with the absorption of oral drugs. Treatment failure due to inadequate itraconazole serum concentrations has been reported in patients with mucositis and poor gastrointestinal absorption (19). Better absorbed formulations, such as the itraconazole-cyclodextrin formulation, will likely be necessary before we can determine the precise role of itraconazole in the treatment of granulocytopenic patients with invasive fungal infections.

Amphotericin B is still the drug of choice for the treatment of most severe systemic fungal infections in immunocompromised patients. For two decades we have come to the conclusion that the toxicities of amphotericin B were inevitable. While new azole and triazole antifungal drugs were being developed, strategies to reduce toxicity and to increase efficacy of amphotericin B were developed. New formulations were proposed. From expensive formulations such as amphotericin B lipid complex, colloidal dispersion or liposomal preparations (20), to simple inexpensive lipid emulsions (21), data from clinical trials are encouraging but limited. The challenge now is to assess through thoughtfully designed, controlled clinical trials the tolerance, efficacy and cost benefit of these new formulations of amphotericin $B$ with those of the new azole antifungal drugs. We must focus our efforts on the severely immunosuppressed patient populations, in whom the consequences of systemic fungal infections are the most devastating. A parallel can be drawn between our present situation of facing fungal infections in immunocompromised hosts, with that of the late 1960s and early 1970s of facing bacterial infections in the same patient populations. The advent of new and more powerful antibacterials led at that time to important clinical trials that contributed to a dramatic decrease in morbidity and mortality due to bacterial infection in these patients. Now that there is a variety of promising systemic antifungal drugs available, the time has come to determine their exact role in the management of immunosuppressed patients. It appears that real progress in systemic antifungal therapy has begun.

\section{REFERENCES}

1. Terrelli CL, Hughes CE. Antifungal agents used for deep-seated mycotic infections. Mayo Clin Proc 1992;67:69-91.

2. Polak A, Dixon DM. In vivo/in vitro correlation of antifungal susceptibility testing using 5-flucytosine and ketoconazole as examples of two extremes. In: Fromtling RA, ed. Recent Trends in the Discovery and Evaluation of Antifungal Agents. Barcelona: JR Prous Science Publishers. 1987:45-59.
3. Drugs for Treatment of Fungal Infections. Med Lett 1992:34:13-6.

4. Francis P, Walsh TJ. Evolving role of flucytosine in immunocompromised patients: New insights into safety, pharmacokinetics, and antifungal therapy. Clin Infect Dis 1992: 15:1003-18.

5. Van HV, Piens MA, Archimbaud E, et al. Serum levels of ketoconazole in bone marrow transplant patients. Nouv Rev Fr Hematol 1983:25:241-4.

6. Piscitelli SC, Goss TF, Wilton JH, et al. Effects of ranitidine and sulcrate on ketoconazole bioavailability. Antimicrob Agents Chemother 1991:35:1765-71.

7. Honig PK, Wortham DC, Zamani K, et al. Terfenadine-ketoconazole interaction: Pharmacokinetic and electrocardiographic consequences. JAMA 1993:269:1513-8.

8. Lazar JD, Wilner KD. Drug interactions with fluconazole. Rev Infect Dis 1990;12(Suppl):S327-33.

9. Saag MS, Powderly WG, Cloud GA, et al. Comparison of amphotericin B with fluconazole in the treatment of acute AIDS-associated cryptococcal meningitis. N Engl J Med 1992;326:83-9.

10. Powderly W, Saag M, Cloud GA, et al. A controlled trial of fluconazole or amphotericin B to prevent relapse of cryptococcal meningitis in patients with the acquired immunodeficiency syndrome. N Engl $\mathrm{J}$ Med 1992:326:793-8.

11. Anaissie E, Bodey GP, Kantarjian H, et al. Fluconazole for chronic disseminated candidiasis in patents with leukemia and prior amphotericin B therapy. Am J Med 1991:91:142-50.

12. Wingard JR, Merz WG, Rinaldi MG, et al. Increase in Candida krusei infection in patients with bone marrow transplantation and neutropenia treated prophylactically with fluconazole. N Engl J Med 1991;325:1274-7.

13. Wingard JR, Merz WG, Rinaldi MG, et al. Association of Torulopsis glabrata infections with fluconazole prophylaxis in neutropenic bone marrow transplant patients. Antimicrob Agents Chemother 1993;37:1847-9.

14. Hitchcock CA. Pye GW, Troke PF, et al. Fluconazole resistance in Candida glabrata. Antimicrob Agents Chemother 1993:37:1962-5.

15. Tucker RM, Haq Y, Denning DW, et al. Adverse events associated with itraconazole in 189 patients on chronic therapy. J Antimicrob Chemother 1990;26:561-6.

16. Denning DW, Tucker RM, Hanson LH, et al. Treatment of invasive aspergillosis with itraconazole. Am J Med 1989;86:791-800.

17. Denning DW, Stepan DE, Blume KG, et al. Control of invasive pulmonary aspergillosis with oral itraconazole in a bone marrow transplant patient. J Infect 1992:24:73-9.

18. Meunier F, Aoun M, Gerard M. Therapy for oropharyngeal candidiasis in the immunosuppressed host: A randomized doubled blind study of fluconazole vs ketoconazole. Rev Infect Dis 1990:12:S364-8.

19. Smith DE, Midgley J, Allan M, et al. Itraconazole versus ketoconazole in the treatment of oral and esophageal candidosis in patients infected with HIV. AIDS 1991;5:1367-71.

20. Lopez-Berestein G, Bodey GP, Fainstein V, et al Treatment of systemic fungal infections with liposomal amphotericin B. Arch Intern Med 1989;149:2533-6.

21. Caillot D, Casasnovas O, Solary E, et al. Efficacy and tolerance of an amphotericin B lipid (intralipid) emulsion in the treatment of candidemia in neutropenic patients. J Antimicrob Chemother 1993;31:161-9.

Michel Laverdière MD Université de Montréal and Department of Microbiology-Infectious Diseases, Hôpital Maisonneuve-Rosemont Montreal, Quebec 


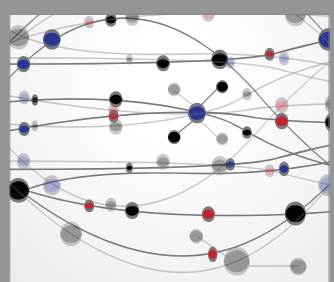

The Scientific World Journal
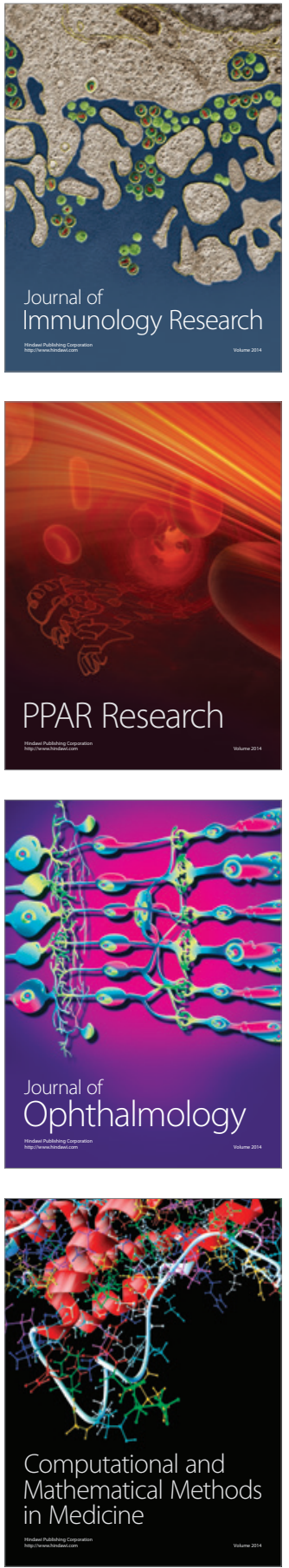

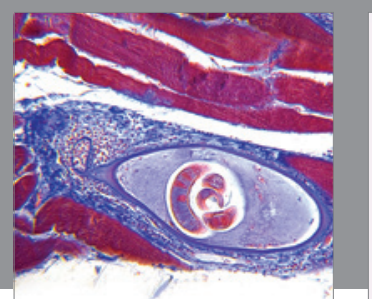

Gastroenterology Research and Practice

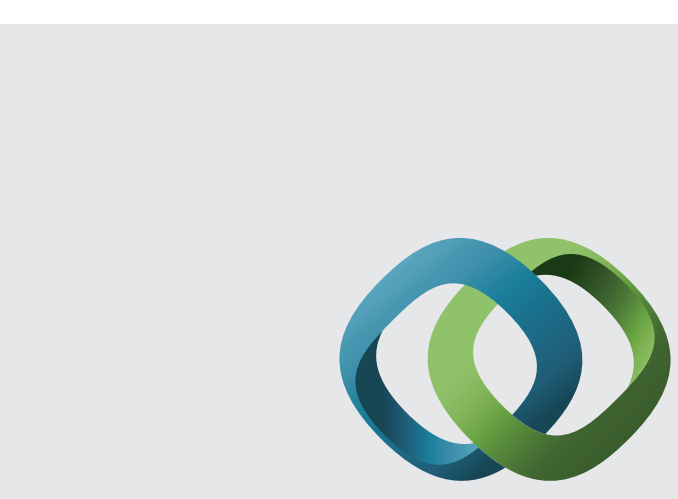

\section{Hindawi}

Submit your manuscripts at

http://www.hindawi.com
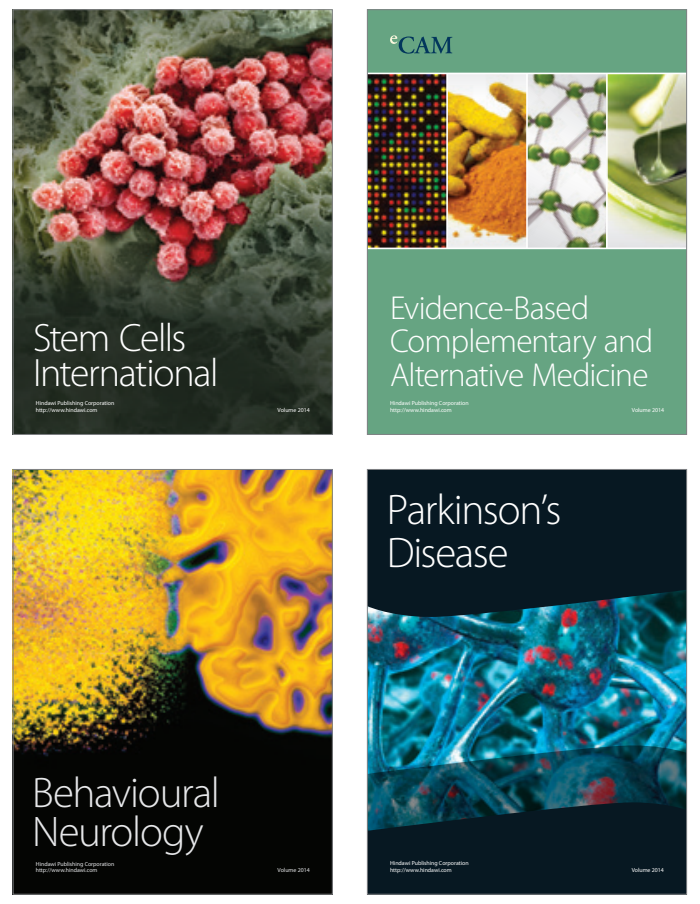
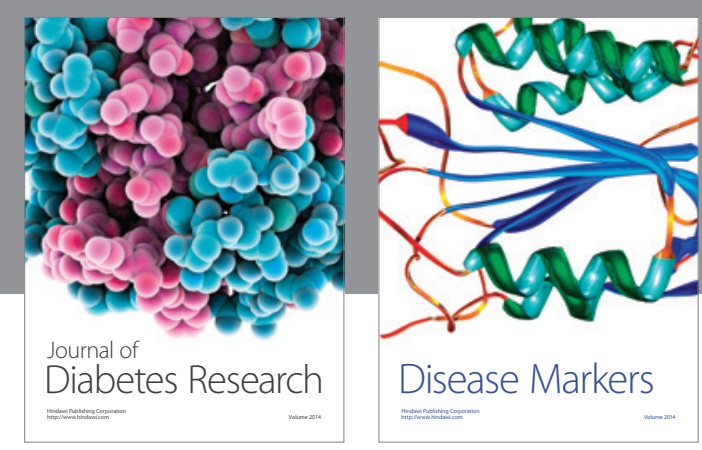

Disease Markers
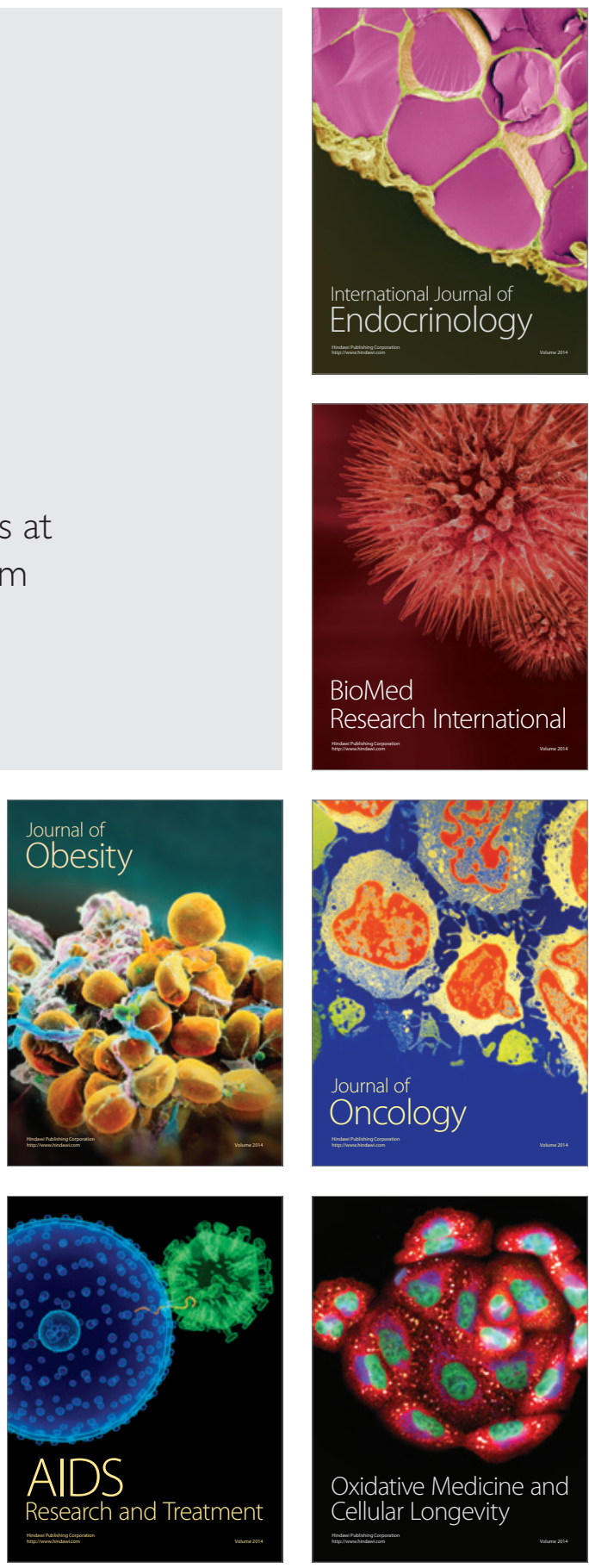